\title{
Report
}

\section{Laminated Magnetic Materials Losses Analysis under Non-Sinusoidal Flux Waveforms in Power Electronics Systems}

D. Aguglia', M. Neuhaus ${ }^{2}$

1 CERN

2 Groupe E S.A.

Keywords: device characterisation, harmonics, measurement, magnetic device, nanotechnology

\begin{abstract}
Losses analyses of laminated magnetic materials subject to non-sinusoidal magnetic flux are presented. Comparative analyses on typical Fe-Si oriented and non-oriented grains and nanocrystalline materials are carried out considering the influence of harmonic phase shift and associated $B-H$ minor loops. Experimental measurements are performed to illustrate the $B-H$ characteristics behaviours. A special experimental effort is given on nanocrystalline material, where Steinmetz coefficients are experimentally identified to analyse the performances of the improved Generalized Steinmetz Equation (iGSE) when phase displaced minor loops occur. It is proven that the iGSE is efficient in predicting nanocrystalline losses versus variable phase displaced harmonics.
\end{abstract}

Presented at:

EPE

Lille, France

September, 2013 


\title{
Laminated Magnetic Materials Losses Analysis under Non-Sinusoidal Flux Waveforms in Power Electronics Systems
}

\author{
Davide Aguglia ${ }^{1}$, and Michel Neuhaus ${ }^{2}$ \\ ${ }^{1}$ CERN-European Organization for Nuclear Research, \\ 1217 Geneva 23, Switzerland \\ Tel. : +41/(0)22 76755 49, E-mail: davide.aguglia@cern.ch \\ ${ }^{2}$ Groupe E S.A., 1763 Granges-Paccot, Switzerland \\ Tel.: +41/(0)26 35254 52, E-mail:michel.neuhaus@groupe-e.ch
}

\section{Keywords}

«Device characterisation», «Harmonics», «Measurement», «Magnetic device», «Nanotechnology».

\begin{abstract}
Losses analyses of laminated magnetic materials subject to non-sinusoidal magnetic flux are presented. Comparative analyses on typical $\mathrm{Fe}-\mathrm{Si}$ oriented and non-oriented grains and nanocrystalline materials are carried out considering the influence of harmonic phase shift and associated $B-H$ minor loops. Experimental measurements are performed to illustrate the $B-H$ characteristics behaviours. A special experimental effort is given on nanocrystalline material, where Steinmetz coefficients are experimentally identified to analyse the performances of the improved Generalized Steinmetz Equation (iGSE) when phase displaced minor loops occur. It is proven that the iGSE is efficient in predicting nanocrystalline losses versus variable phase displaced harmonics.
\end{abstract}

\section{Introduction}

In power electronics systems, components such as filtering inductors, distribution or pulse transformers, and rotating electrical machines are generally composed of laminated magnetic materials and are subject to non-sinusoidal voltage waveforms. The prediction of magnetic losses in these elements is essential if one considers the growing efforts of the designer in optimizing volumes and efficiencies and selecting the right, cost effective, laminated material. In this paper the usable analytical formulation for losses calculation in the non-sinusoidal case is presented. Afterward a set of experimental illustrations of several $B-H$ curves is presented in order to show the influence of different voltage, or magnetic flux, shapes. In particular, an experimental evaluation of the influence of harmonic phase displacement, producing magnetic minor loops, is presented. Finally, experimental losses measurements on nanocrystalline material are compared with the iGSE analytical formulation, showing its capabilities in predicting losses in the general non-sinusoidal case with and without the presence of minor loops in the $B-H$ characteristic. The materials under tests are oriented and nonoriented grain Fe-Si 3\% and Nanocrystalline.

\section{Magnetic losses analytical prediction}

Magnetic losses are produced by the dissipation of an electrical current circulating in the magnetic material [1]. Supposing the knowledge of the current density space-time distribution $j(r, t)$ in the material, one could simply derive the power loss per cycle $P / f$ as expressed in (1).

$$
\frac{P}{f}=\frac{1}{V} \iiint_{V} \int_{0}^{\frac{1}{f}} \frac{|j(r, t)|^{2}}{\sigma} d t d^{3} r
$$


where $\sigma$ is the electric conductivity, $V$ the volume and $f$ the frequency. For convenience, electrical engineers are used to separate this power loss into three types: hysteresis loss $P_{h y s}$, eddy current loss $P_{e d}$, and excess loss $P_{e x c}[2]$. This separation does not derive from a physical interpretation of three different phenomena; however, this comes from a distinction in terms of different time and space scales behaviours of the same physical phenomena. Thus, the total losses density in a ferromagnetic material becomes:

$$
P_{t o t}=P_{h y s}+P_{e d}+P_{e x c}
$$

Several mathematical models have been proposed in the literature for predicting magnetic losses. A review of these models is presented in [3]. Hysteresis models methods, such as the Jiles-Atherton [4] or the Preisach [5] ones can be used for deriving accurate results. However, they are limited in their practical use, due to the very high number of empirical parameters which have to be determined with a very strong experimental effort. The most usable formulation comes from the well-known empirical equation from the work of Steinmetz [6], which combined with the latest approaches in losses separation of (2), leads to the expression reported in (3).

$$
\begin{aligned}
& P_{\text {tot }}=P_{\text {hys }}+P_{e d}+P_{e x c}=a f \hat{B}^{x}+b f^{2} \hat{B}^{2}+c f^{1.5} \hat{B}^{1.5}=C_{m} f^{\alpha} \hat{B}^{\beta}+C_{2} f^{2} \hat{B}^{2} \\
& \text { with } C_{2}=\frac{\pi^{2}}{6} \frac{t_{l}}{\sigma \cdot d_{v}}
\end{aligned}
$$

Where $a, b, c, C_{m}, \alpha$ and $\beta$ are constants which depend on the material characteristics, $C_{m}$ is generally provided by the material supplier, $x$ is the Steinmetz coefficient, $t_{l}$ is the lamination thickness and $d v$ the magnetic material volumetric density [7]. A set of Steinmetz coefficients can be found in [8]. For a complete description of the losses separation in (3) one can refer to [1] or, for a summary, to [9]. The drawback of the Steinmetz based empirical formulation in (3) comes from the assumption of a sinusoidal time variation of magnetic flux. Unfortunately this hypothesis makes the expression in (3) not usable for power electronics magnetic devices due to magnetic flux harmonics content. For this reason Authors in [10] proposed a modified version of the Steinmetz formulation, based on the general hypothesis for instantaneous core loss presented in [11], in order to extend it to the non-sinusoidal case, by considering the property of losses being dependent to the magnetic induction rate of change $d B / d t$. This modified version has been even further improved in [12], and the result is given in (4) and (5).

$$
P_{t o t}=\frac{1}{T} \int_{0}^{T} k_{i}\left|\frac{d B}{d t}\right|^{\alpha}(\Delta B)^{\beta-\alpha} d t
$$

with:

$$
k_{i}=\frac{C_{m}}{(2 \pi)^{\alpha-1} \int_{0}^{2 \pi}|\cos \theta|^{\alpha} 2^{\beta-\alpha} d \theta}
$$

where $\Delta B$ is the induction excursion, or peak-to-peak value, during one cycle. Equations (4) and (5) constitute the well-known improved Generalized Steinmetz Equation (iGSE). Notice that in (4) one computes the average rate of change of the magnetic induction during one cycle (integral term). In (4) and (5) one only uses Steinmetz parameters which are always obtainable from materiel manufacturer, supplier, or easily identified from experimental measurements. Furthermore, as stated in [12], this model ((4) and (5)) is suitable for predicting losses in presence of minor loops in the $B-H$ characteristic, if the associated peak-to-peak induction excursion of each minor loop $\Delta B_{\text {mloop }}$ is known. Many other formulations have been presented, however, due to its better accuracy [13], only the iGSE is retained in this paper. 


\section{Experimental measurement method for losses analyses}

The cores under test are equipped with two identical windings, made of Litz wire, to form two identical transformers with a unity ratio between primary and secondary. The three different examined materials are:

1. Non-Oriented Grain, $0.35 \mathrm{~mm}$ lamination thickness (type NO2735).

2. Oriented Grain, $0.23 \mathrm{~mm}$ lamination thickness (type GOM3X).

3. $\mathrm{FeSiB}+\mathrm{NbCu}$ Nanocrystalline, $20 \mu \mathrm{m}$ tape thickness (Type VITROPERM 500F - no core-cut).

A measurement method is based on the schematic of Fig. 1. Schematic of Fig. 1b) is used for tests with perfectly square shaped voltage supply, whereas schematic of Fig. 1a) is used for harmonic injection thanks to a high frequency linear power amplifier. Along with geometrical dimensions, the induced secondary voltage is used to determine the magnetic induction $B$, and the measured primary current $i_{l}$ allows determining the magnetic field strength as shown in (6) and (7).

$$
\begin{aligned}
& B(t)=\frac{1}{S \cdot N_{2}} \int V_{2} d t+C \\
& H(t)=\frac{N_{1} i_{1}(t)}{l_{m}}
\end{aligned}
$$

where $C$ is the magnetic induction initial condition (which can be set to 0 if the integration starts at $\pi / 2 \mathrm{rad}$ of phase displacement from the zero crossing of the sinusoidal induced voltage), and $l_{m}$ and $S$ are the core mean length of the magnetic flux path and the iron cross section area respectively. The losses are measured via a high frequency and high accuracy power meter (with auto adaptive input ranges). Fig. 2 shows the experimental set-up and the measured sinusoidal $B-H$ characteristics of the three materials under test. Notice how even at $10 \mathrm{kHz}$, compared to $50 \mathrm{~Hz}$ for Fe-Si materials, nanocrystalline material presents a much better characteristic.

a)

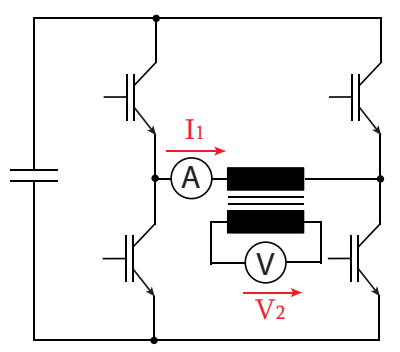

b)

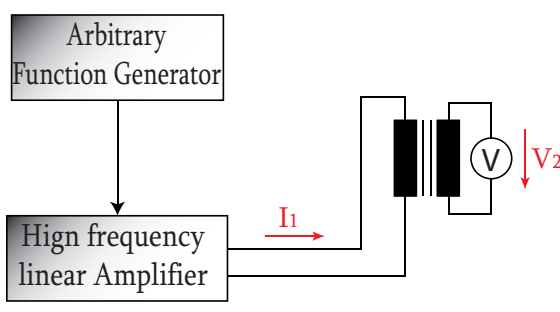

Fig. 1: Tests set-ups schematics for $B-H$ curves and losses measurements - a) via IGBT H-Bridge for voltage square waves production; b) via high frequency linear amplifier for harmonics injection.
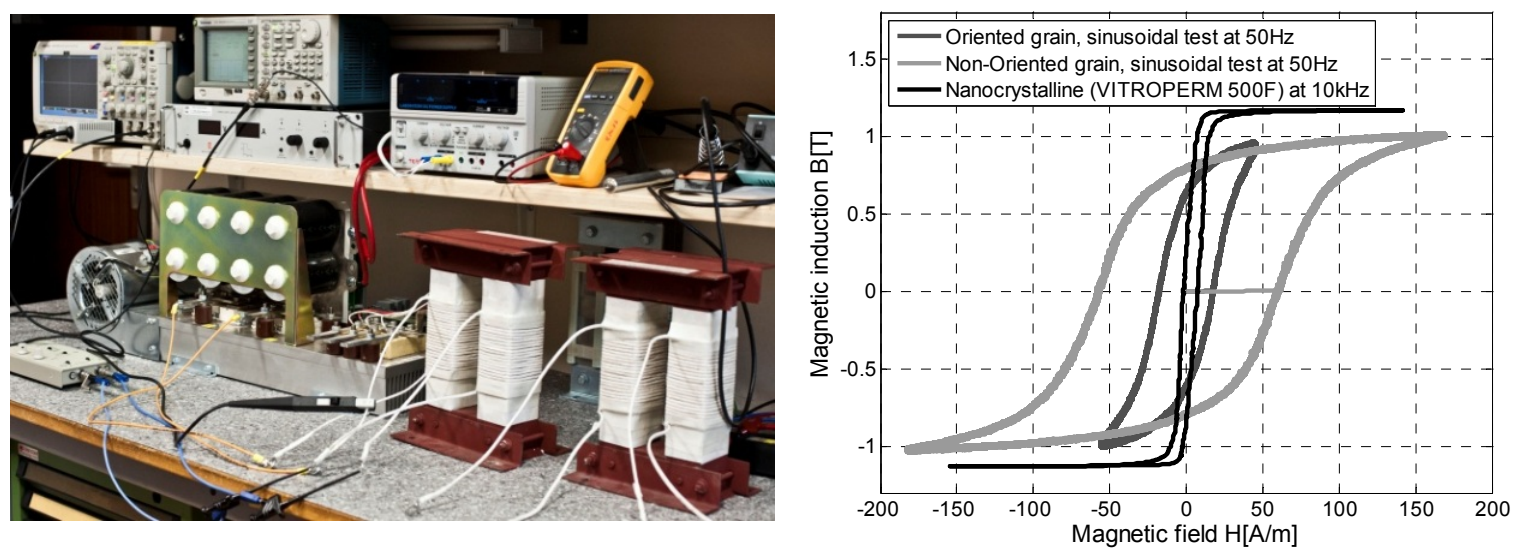

Fig. 2: Left - Experimental set-up in the configuration of Fig. 1a); Right $-B-H$ characteristics of the two Fe-Si materials at $50 \mathrm{~Hz}$ and of Nanocrystalline at $10 \mathrm{kHz}$. 


\section{Materials comparison and voltage harmonics phase displacement analysis}

\section{B-H characteristics analyses under non-sinusoidal excitation}

In many power electronics applications, the voltage shape applied to magnetic component (e.g. transformers, inductors and motors) differs from the sinusoidal one; therefore the magnetic flux and induction have a non-sinusoidal time variation. To analyse the losses of the general non-sinusoidal case, it is worth to illustrate the effect of different non-sinusoidal magnetic fluxes on the $B-H$ characteristic of several magnetic materials. This analysis is of great importance since magnetic losses are directly linked to the surface area described by the $B-H$ characteristic (8).

$$
P=V \cdot f \oint H \cdot d B
$$

In several applications rectangular shaped voltages are applied across filtering inductances or pulse transformers. Figure 3 presents the measured $B-H$ characteristics of each material under test for sinusoidal and rectangular shaped voltage supplies, which correspond to sinusoidal and triangular magnetic induction time variation respectively. For each magnetic material, and for the same maximal induction, notice how the $B-H$ characteristics are shrunk for the rectangular voltage shape case. Considering (8), this means that losses are reduced in the rectangular voltage shape case. It is also known that the $B-H$ characteristic presents a "growing" effect for increasing sinusoidal frequencies (higher losses at higher frequencies as described by the ordinary Steinmetz equation). All these observations agree with the iGSE theory in (4) and (5), where losses are proportional to the average magnetic induction rate of change $1 / T \cdot \int|d B / d t|^{\alpha}$. Indeed the average rate of change of a triangular induction waveform (rectangular voltage shape) is lower than a sinusoidal one, and clearly a higher frequency sinusoidal induction waveform has higher average rate of change than a lower frequency one. To generalize the study one must consider the general case where voltage harmonics can be injected with arbitrary phase shifts. For this validation experimental measurements have been carried out on the oriented grain material considering a phase sweep of the $3^{\text {rd }}$ voltage harmonic applied to the system presented in Fig. 1 b), and results are presented in Fig. 4 (for the case where the $3^{\text {rd }}$ harmonic amplitude is equivalent to the fundamental one). Notice how in Fig. 4 the minor loops are displaced with respect to the fundamental $B-H$ loop, where a change of losses should appear. Furthermore, notice that no minor loops are created below a certain amount of harmonic phase displacement.

(a)

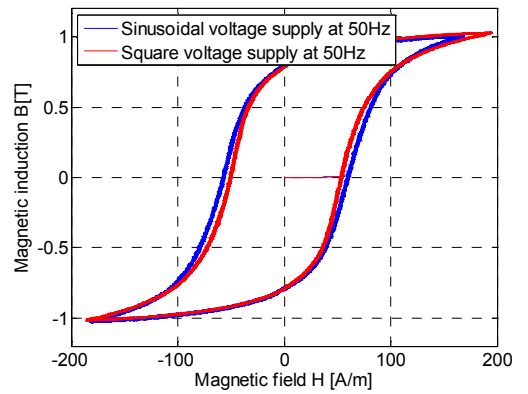

(b)

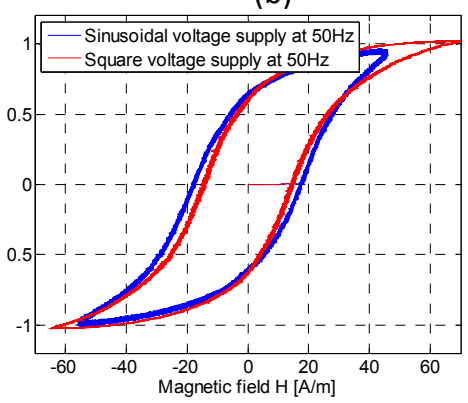

(c)

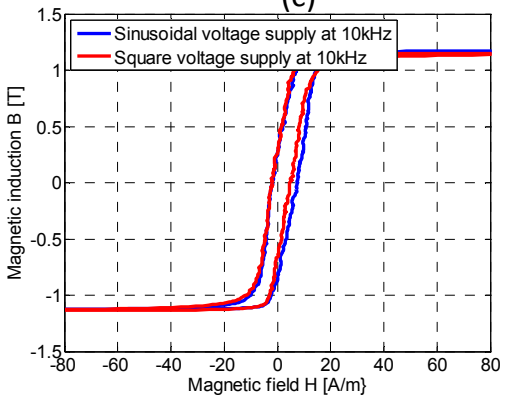

Fig. 3: Measured $B-H$ characteristics of a) NO2735, b) GOM3X, and c) Nanocrystalline, for sinusoidal and squared voltage supplies.

(a)

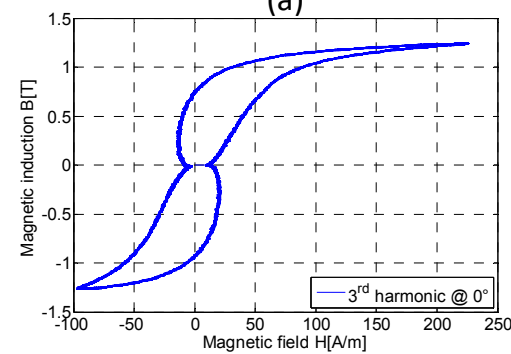

(b)

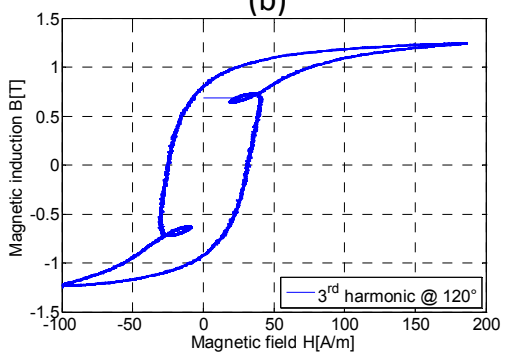

(c)

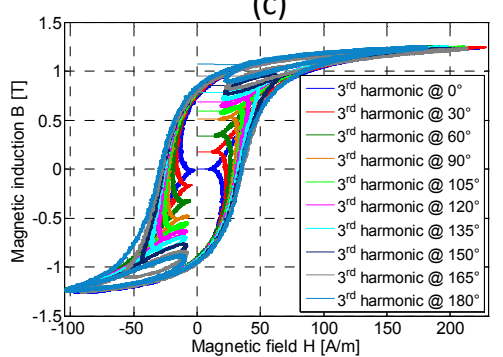

Fig. 4: $3^{\text {rd }}$ voltage harmonic with phase displacement effect on the $B-H$ loop of the GOM3X material. Fundamental frequency at $50 \mathrm{~Hz}$ and $3^{\text {rd }}$ harmonic and fundamental with same amplitude. 
The effect of a third harmonic phase displacement has been measured on nanocrystalline material as well and is presented in Fig. 5. Figures 5 (a), (b), and (c) results from a third harmonic injection with same amplitude as the fundamental one, whereas Fig. 5 (d), (e), and (f) derive from a third harmonic injection with half of the fundamental amplitude. For the sake of comparison Fig. 5 (e) presents the $B$ $H$ characteristics of a pure sinusoidal supply and with third harmonic injection. For all these measurements the peak amplitude of the voltage supply has been selected in order to respect exactly the same maximal induction $B$ for every harmonic phase displacement.

Depending on amplitudes and phase displacements of the injected harmonic, minor loops may appear. In this case losses computation becomes slighly more complicated, since the contribution of each loop (major and minor) must be considered. This means that the actual $B-H$ surface area to be considered for losses computation in presence of minor loops includes the area described by the fundamental, or major loop, in addition to the areas contributions of each of the minor loops. The iGSE can be used to estimate the losses in presence of minor loops by computing the average induction rate of change for each loop. The Authors of this procedure, described in [12], programmed a routine available in [14] which will be used in the last section.

\section{Steinmetz coefficients identification for nanocrystalline material}

In order to evaluate the efficiency of the iGSE subject to phase displaced induction harmonics, coefficients of the Ordinary Steinmetz Equation (OSE) have been experimentally identified on nanocrystalline VITROPERM $500 F$ material.

Figure 6 presents the measurement results along with the estimations from the OSE with identified coefficients in (9).

$$
P_{d}=K f^{\alpha} \hat{B}^{\beta}=1.19 e^{-6} f^{1.747} \hat{B}^{2.19}[W / k g]
$$

(a)

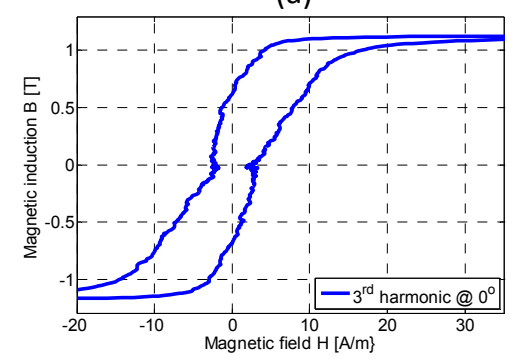

(d)

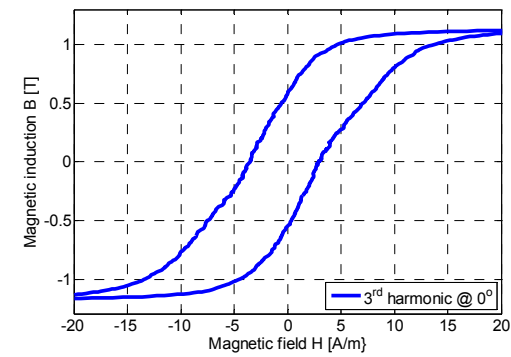

(b)

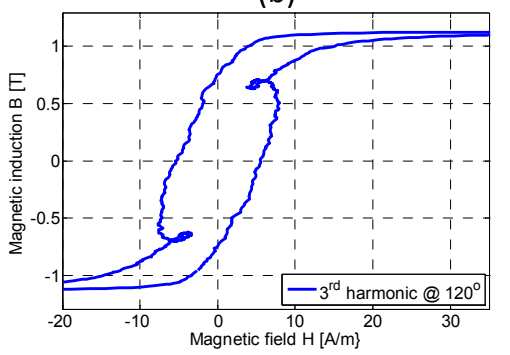

(e)

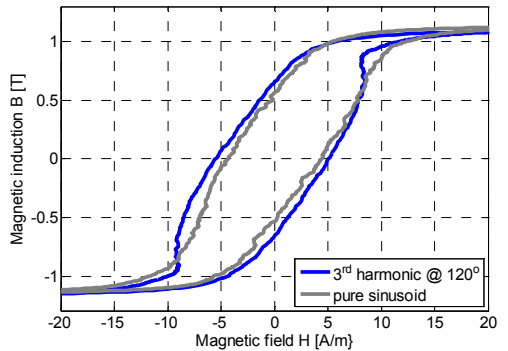

(c)

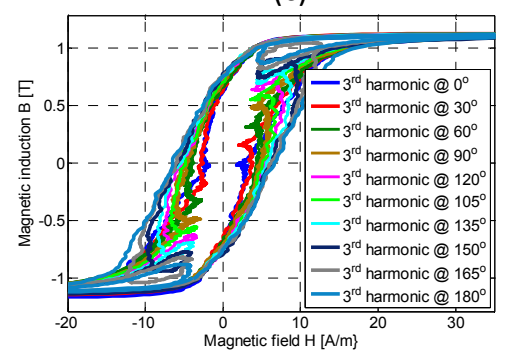

(f)

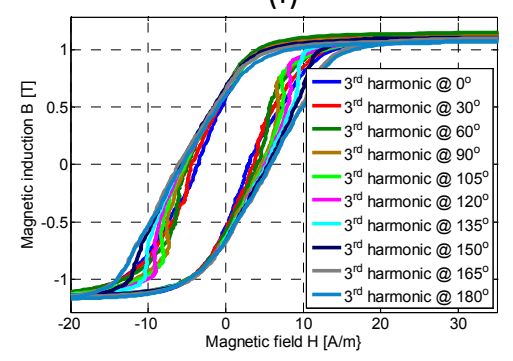

Fig. 5: a), b) and c) $3^{\text {rd }}$ voltage harmonic with phase displacement effect on the $B-H$ loop of the Nanocrystalline material. Fundamental frequency at $10 \mathrm{kHz}$ and $3^{\text {rd }}$ harmonic amplitude equal to the fundamental. (d) (e) and (f) $3^{\text {rd }}$ harmonic amplitude equal to half the fundamental one. 


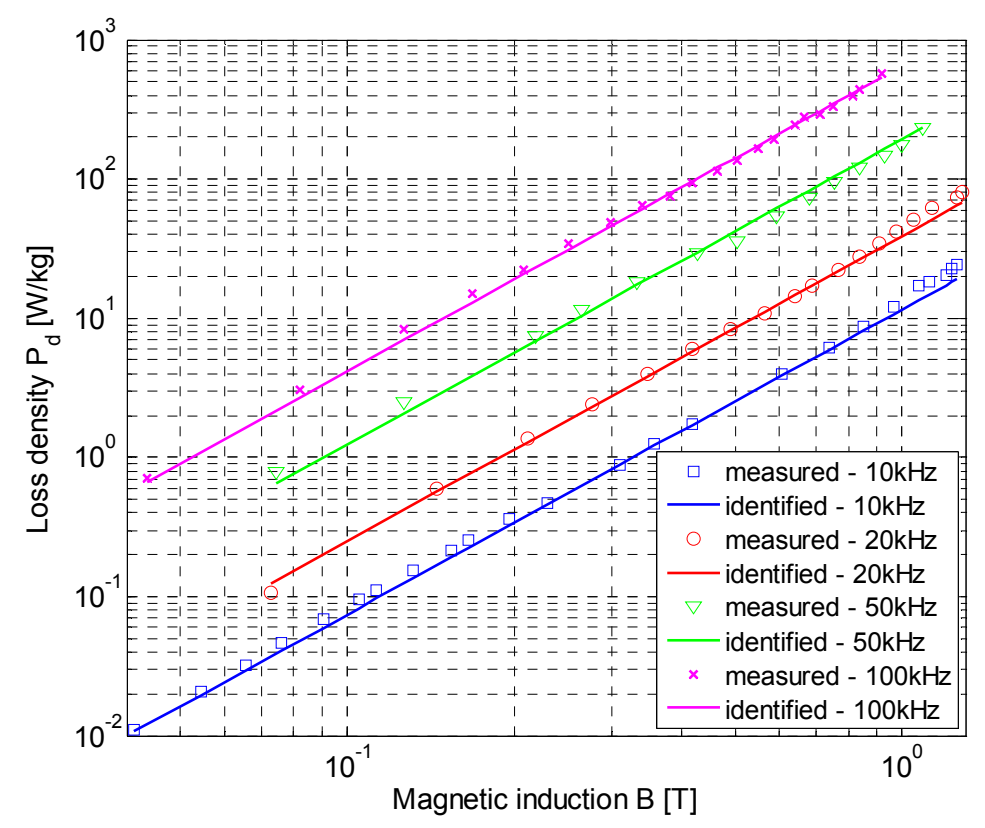

Fig. 6: Measured losses on VITROPERM 500F nanocrystalline material (no core-cut) for Steinmetz coefficient identification purposes.

\section{Losses analysis versus variable phase displaced harmonics injection}

In the literature no experimental validations have been carried out verifying the validity of the iGSE model presented in [10] and [12] (4), for phase displaced harmonics. This analysis has been previously presented for ferrite materials in [15] where the GSE was firstly proposed. In [12] the iGSE formulation in (4) has been validated for one case only, where the phase displacement of the added magnetic flux third harmonic was null. For illustration purposes the losses versus voltage phase displacement for the $3^{\text {rd }}$ and $5^{\text {th }}$ harmonics have been measured for several conditions of maximal induction and fundamental frequencies on the oriented grain material. Results are reported in Fig. 6 . One can notice how losses are increasing with voltage harmonic phase displacement, which is in agreement with the tendency noticeable in the measured $B-H$ characteristics of Fig. 4 . The same losses characteristics have been measured on the nanocrystalline material and are presented in Fig. 7. Measurements are carried out at $10 \mathrm{kHz}$ of fundamental frequency and third harmonic injection with fundamental $\left(A_{h}=A_{f}\right)$ and half of fundamental $\left(A_{h}=A_{f} / 2\right)$ amplitude.
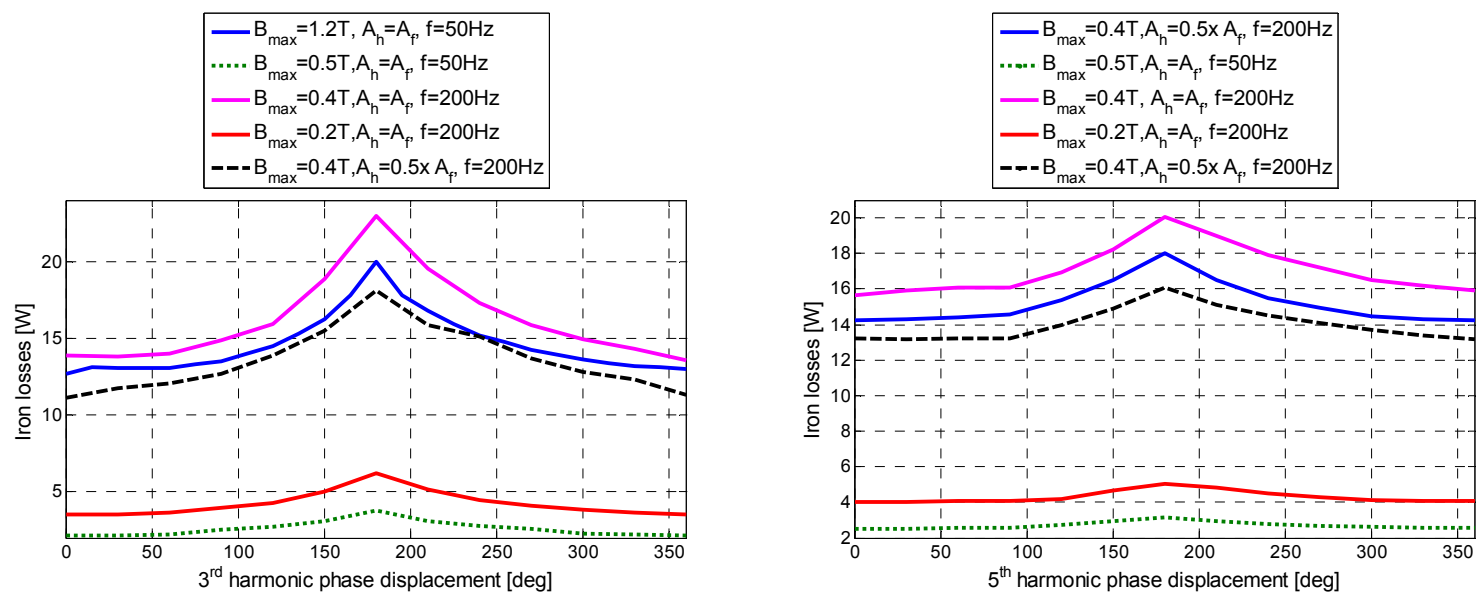

Fig. 6: GOM3X measured absolute iron losses versus $3^{\text {rd }}$ and $5^{\text {th }}$ harmonics phase displacement for different fundamental frequencies and harmonic amplitudes. 


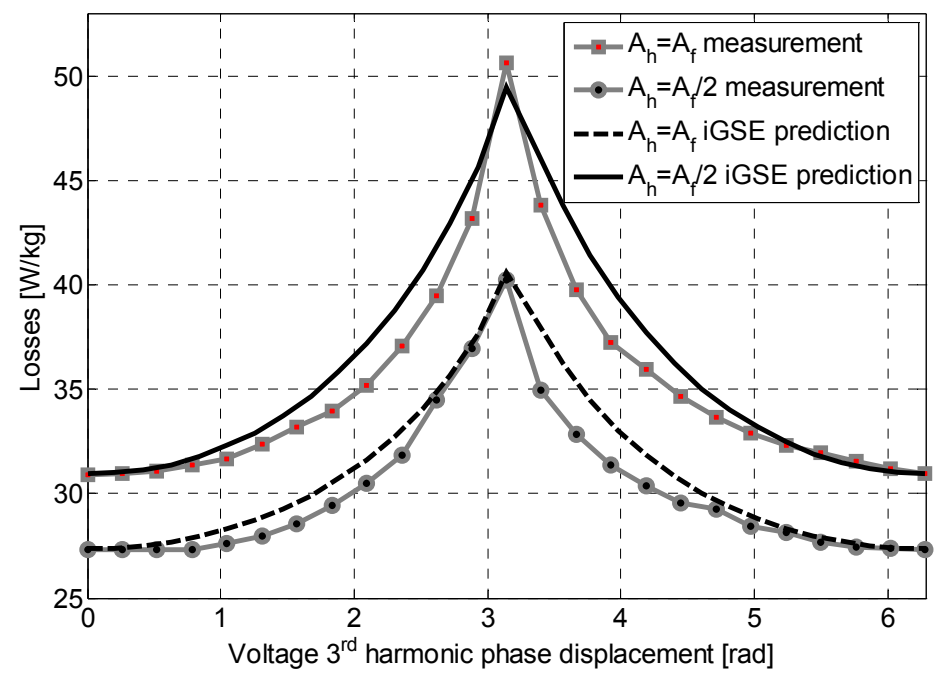

Fig. 7: Nanocrystalline measured iron losses density versus $3^{\text {rd }}$ harmonics phase displacement for two harmonic amplitudes. Fundamental frequency at $10 \mathrm{kHz}$.

The identified Steinmetz coefficients on the nanocrystalline have been used in the iGSE formulation to estimate losses in presence of the phase displaced voltage third harmonics. Results are compared with measurements in Fig. 7. In the case where $A_{h}=A_{f} / 2$ no minor loops are created, as shown in Fig. 5(f), whereas when $A_{h}=A_{f}$ minor loops are present. The iGSE gives quite accurate results when phase displacing voltage harmonics to the fundamental, proving that this formulation is actually well founded on solid physical interpretation of losses in magnetic materials. In the measurements of Fig. 7 special attention has been given in keeping a constant maximal induction for each phase displacement. That is why working in deep saturation is convenient in order to guarantee a constant maximal induction (corresponding $B-H$ curves in Fig. 5). However, Fig. 7 shows an over estimation of losses from the iGSE, except for voltages phase displacements of $0 \mathrm{rad}$ and $\pi \mathrm{rad}$. Nevertheless the error seems to never go beyond 20\%, which considering measurements errors in the Steinmetz coefficients identification process and errors in the losses direct measurements of Fig. 7, may be more than acceptable. Furthermore the iGSE seems to slightly over estimate losses, which may be accepted from designers. It has to be noted that the nanocrystalline material tested in this paper is not a cut-core. As presented in [16] the preparation for producing cut-cores highly modifies the magnetic properties of the core losses, leading to different Steinmetz coefficients. Particularly, cut-cores always present more losses than entire, not cut, ribbon bobbins.

\section{Conclusion}

Effects on the $B-H$ characteristic and core losses under sinusoidal and non-sinusoidal magnetic inductions have been presented for Fe-Si and nanocrystalline materials. The iGSE has been verified on nanocrystalline materials for losses calculation in case of phase displacements of injected harmonics in the excitation voltage. Compared with the GSE [15] it is demonstrated that the iGSE gives better results in the whole range of phase displaced harmonics. This further demonstrates the physical validity of the iGSE formulation and an acceptable accuracy when applied to nanocrystaline materials.

\section{References}

[1] G. Bertotti, "Hysteresis in magnetism," Academic Press, Inc., 1998, 543 p.

[2] W. A. Roshen, "A Practical, accurate and very general core loss model for nonsinusoidal waveforms," IEEE Trans. Power Electr. Vol. 22, No. 1, 2007, pp. 30 - 40

[3] Krings A., Overview and comparison of iron loss models for electrical machines, Journal of Electrical Engineerig, Vol. 10 no 3, 2010, pp. 162-169 
[4] D. C. Jiles, D. L. Atherton, “Theory of ferromagnetic hysteresis,” J. Appl. Phys. Vol. 55, No. 6, 1984, pp. $2115-2120$

[5] S. Y. R. Hui, J. Zhu, "Magnetic hysteresis modelling and simulation using the Preisach theory and TLM technique," in Proc. IEEE PESC, 1994, pp. 837 - 842

[6] C. P. Steinmetz, "On the law of hysteresis," AIEE Transactions, Vol. 9, No. 1, 1892, pp. 3 - 64

[7] M. Popescu, D. Ionel, "A Best-Fit Model of Power Losses in Cold Rolled-Motor Lamination Steel Operating in a Wide Range of Frequency and Magnetization”, IEEE Transactions On Magnetics, vol. 43, Issue 4, 2007, pp. 1753-1756

[8] Akiror J. C., Pillay P., On the coefficients of core loss formulas for electrical machines, IECON 2012, pp. $1927-1933$

[9] A. Boglietti, A. Cavagnino, M. Lazzari, M. Pastorelli, "Predicting Iron Losses in soft Magnetic Materials with Arbitrary Voltage Supply: An Engeneering Approach,” IEEE Trans. Magn., Vol. 39, No. 2, 2003, pp. $981-988$

[10] J. Reinert, A. Brockmeyer, R. A. A. De Doncker, "Calculation of losses in ferro- and ferromagnetic materials based on the modified Steinmetz Equation," IEEE Trans. Ind. Appl., Vol. 37, No. 4, 2001, pp. $1055-1061$

[11] Lancarotte M. S., Penteado Jr. A. A., Estimation of core losses under sinusoidal or non-sinusoidal induction by analysis of magnetization rate, IEEE Transactions on Energy Conversion, Vol. 16 no. 2, June 2001, pp. $174-179$

[12] K. Venkatachalam, C. R. Sullivan, T. Abdallah, H. Tacca, "Accurate prediction of ferrite core loss with nonsinusoidal waveforms using only Steinmetz parameters," in Proc. IEEE Workshop Comput. Power Electron., 2002, pp. $36-41$

[13] Villar I., Global loss evaluation method for nonsinusoidally fed medium-frequency power transformers, IEEE Transactions on Industrial Electronics, Vol. 56 no 10, 2009, pp. 4132-4140

[14] Dartmouth Magnetic Component Research Website: http://engineering.dartmouth.edu/inductor/index.shtml

[15] Li, J., Adballah T., Sullivan C. R., Improved calculation of core loss with nonsinusoidal waveforms, $36^{\text {th }}$ IEEE Industry Applications Society (IAS) annual meeting, Vol. 4, 2001, pp. 2203 - 2210

[16] Shen, W., Wang F.,Boroyevich D., Tipton C. W., Loss characterization and calculation of nanocrystalline cores for high-frequency magnetics applications, IEEE Transactions on Power Electronics, Vol. 23 no 1, 2008 , pp. $475-484$ 